\title{
Perceived Parental Attitudes of Gender Expansiveness: Development and Preliminary Factor Structure of a Self-Report Youth Questionnaire
}

\author{
Marco A. Hidalgo, ${ }^{1-3, *}$ Diane Chen, ${ }^{1-3}$ Robert Garofalo, ${ }^{1,3}$ and Catherine Forbes ${ }^{4}$
}

\begin{abstract}
Purpose: Parental acceptance of gender identity/expression in lesbian, gay, bisexual, transgender, and queer/ questioning (LGBTQ+) youth moderates the effects of minority stress on mental health outcomes. Given this association, mental health clinicians of gender-expansive adolescents often assess the degree to which these youth perceive their parents/primary caregivers as accepting or nonaffirming of their gender identity and expression. While existing measures may reliably assess youth's perceptions of general family support, no known tool aids in the assessment an adolescent's perceived parental support related to adolescent gender-expansive experiences. Methods: To provide both clinicians and researchers with an empirically derived tool, the current study used factor analysis to explore an underlying factor structure of a brief questionnaire developed by subject-matter experts and pertaining to multiple aspects of perceived parental support in gender-expansive adolescents and young adults. Respondents were gender-expansive adolescents and young adults seeking care in an interdisciplinary gender-health clinic within a pediatric academic medical center in the Midwestern United States.

Results: Exploratory factor analysis resulted in a 14-item questionnaire comprised of two subscales assessing perceived parental nonaffirmation and perceived parental acceptance. Internal consistency and construct validity results provided support for this new questionnaire.

Conclusion: This study provides preliminary evidence of the factor structure, reliability and validity of the Parental Attitudes of Gender Expansiveness Scale for Youth (PAGES-Y). These findings demonstrate both the clinical and research utility of the PAGES-Y, a tool that can yield a more nuanced understanding of family-related risk and protective factors in gender-expansive adolescents.
\end{abstract}

Keywords: transgender; parental support; factor analysis; adolescents; measure development

\section{Background}

Parental support, including providing emotional support, ensuring safety, and validating personal experiences, positively impacts adolescent development and may counteract the deleterious effects of adverse childhood experiences. ${ }^{1,2}$ Lesbian, gay, bisexual, transgender, and queer/questioning (LGBTQ+) adolescents are particularly vulnerable to discrimination and victimization on account of their sexuality, gender identity, and/or gender expression. ${ }^{3}$ These adverse experiences have been linked to increased odds of psychopathology, suicidality, substance use, homelessness, and sexual risk behavior. ${ }^{4-9}$ LGBTQ+ adolescents who perceive their parents to be supportive of their sexual or gender identities report better psychosocial health and health behavior outcomes than LGBTQ+ adolescents without supportive parents. ${ }^{10-12}$

Examining parental support in gender-expansive (e.g., transgender, gender-nonconforming) adolescents

\footnotetext{
'Division of Adolescent Medicine, Ann \& Robert H. Lurie Children's Hospital of Chicago, Chicago, Illinois.

${ }^{2}$ Department of Psychiatry and Behavioral Sciences, Northwestern University, Feinberg School of Medicine, Chicago, Illinois.

${ }^{3}$ Department of Pediatrics, Northwestern University, Feinberg School of Medicine, Chicago, Illinois.

${ }^{4}$ Division of Adolescent and Young Adult Medicine, Center for Transyouth Health and Development, Children's Hospital Los Angeles, Los Angeles, California.
} 
is especially important as they experience lower levels of social support and general health than their cisgender counterparts, including lesbian, gay, and bisexual youth. ${ }^{13,14}$ When they experience parental rejection or insufficient support, gender-expansive youth are likely to face economic and societal marginalization, incarceration, and physical abuse, leaving them at significantly higher risk for drug abuse, violence, HIV acquisition, other sexually transmitted infections, and homelessness as they age. ${ }^{15-18}$

Degrees of parental acceptance and rejection exist along two separate spectra and can, therefore, both be expressed by a parent. ${ }^{19,20}$ For example, a parent may be somewhat accepting of their child's disclosure of transgender identity, but prohibit (or not fully support) their child from initiating a social gender transition (e.g., using an affirmed name or gender pronouns) in or outside the home. In other cases, parents viewed as otherwise supportive of an adolescent's gender identity, and expression may be perceived as rejecting if they refuse to support an adolescent's desire to pursue gender-affirming hormone therapy and consent for treatment. Parental rejection may result from a parent's doubts about the validity of their child's selfidentified gender or due to deficits in parenting ability or capacities. ${ }^{21}$ Degrees of parental rejection may also be informed by parents' faith-based beliefs, negative views of diversity in sexual and gender identity, or a lack of information on gender-affirming medical interventions. ${ }^{10,19,22}$

Although limited, extant literature on parental support of gender-expansive adolescents confirms its protective potential, when present. ${ }^{22}$ Forms of parental support reported by gender-expansive adolescents include acceptance and affirmation of gender identity, ${ }^{23}$ advocacy, and assuring safety from discrimination or victimization. ${ }^{24}$ One recent study of transfeminine adolescents found that parental acceptance of genderexpansive identity was associated with lower rates of adolescent psychological distress. ${ }^{23}$ Gender-expansive youth, compared with other socially marginalized youth, are vulnerable to school-based and communitylevel victimization on account of their gender identity and/or expression. Gender-expansive adolescents have described parental acceptance as including parents advocating on their behalf (e.g., extended family and school settings) or protecting them from discrimination. $^{24}$

In summary, research on LGBTQ+ youth, including some pertaining to gender-expansive adolescents, has underscored the protective nature of parental acceptance, the potentially deleterious impact of parental rejection, and co-occurrence of both parental acceptance and rejection. Mental health clinicians treating genderexpansive adolescents and their families commonly assess these important aspects of social support. ${ }^{25-27}$ Although employed in research on LGBTQ+ youth, psychometrically sound and widely used measures of social support, such as the Multidimensional Scale of Perceived Social Support (MSPSS), ${ }^{28}$ assess general family support and not parental support related to a child's gender expansiveness. However, no known tool currently exists to aid clinicians in globally assessing parental support for gender diversity as reported by gender-expansive youth.

\section{Measuring parental support in gender-expansive adolescents}

Several attempts have been made to construct a measure of parental attitudes regarding gender expansiveness in youth, particularly parental support. One such measure was developed using data from qualitative interviews of LGBTQ+ adolescents. ${ }^{10}$ In this study, researchers constructed a multi-item measure of parental acceptance using a four-point scale addressing the frequency of several accepting behaviors. For example: "How often did any of your parents/caregivers appreciate your clothing or hairstyle even though it might not have been typical for your gender?" Scores from the measure were then divided to indicate low, moderate, or high levels of parental acceptance. This measure has not yet been published or made available for use by researchers or clinicians. Another measure, developed for use with transfeminine adolescents, consisted of a 10-item dichotomous (yes/no) response scale to items such as, "Did any of your parents/caregivers ever talk about your trans identity with you?" 23 Although this measure was found to have high internal consistency with a sample of transfeminine adolescents, its dichotomous scores fail to capture potential variability in degree of parental acceptance experienced by the sample. In addition, it may prove to be less valid when used with other populations of gender-expansive youth (e.g., transmasculine, nonbinary).

The two measures highlighted in this study assess perceived parental attitudes toward gender identity and parental support of gender expression. However, no known questionnaire assesses other relevant aspects of perceived parental support in gender-expansive adolescents, including degree of parental advocacy and 
parental attitudes regarding gender expansiveness. With the aim of providing both clinicians and researchers with an empirically derived tool, the current study explored the underlying factor structure of a brief questionnaire pertaining to multiple aspects of perceived parental support in gender-expansive adolescents and young adults.

\section{Methods}

Parental attitudes of gender expansiveness scale for youth development

The scale items were codeveloped by two subjectmatter experts (SMEs) to optimize content validity. One SME was a gender-expansive young adult active in transgender youth communities who, serving as a key informant, shared personal and community testimonies that informed the development of items. The second SME was a cisgender female, licensed clinical psychologist who practiced in an adolescent gender clinic within a pediatric academic medical center located in southern California. The SMEs met regularly over the course of a year to develop and revise items based on existing research, personal and community testimony, and clinical wisdom and experience.

A 17-item questionnaire was completed in late 2012 and it aimed to ascertain the degrees to which genderexpansive adolescent respondents' perceived parental attitudes and behaviors to be accepting or rejecting regarding their gender identity/expression. Using a 5-point Likert scale ranging from "strongly disagree" to "strongly agree," participants responded to items such as "My parent(s) have problems with my gender expression," "My parent(s) advocate for my rights as a gender-nonconforming/trans child," and "My parent(s)' religious beliefs help them be supportive of me." Values of 1-5 were assigned to each item, such that a higher score was indicative of greater parental support.

\section{Survey data collection}

Survey data were collected from adolescents as standard of care at their baseline clinical visit along with several other paper-and-pencil self-report measures, including emotional and behavioral symptom checklists and scales pertaining to gender identity and body satisfaction (a comprehensive review of these measures is detailed elsewhere) ${ }^{25}$ Before starting the battery, all questionnaires were described to respondents and parents (for patients who were minors). Participants were told that the 17 items of relevance pertained to "parental attitudes about gender-nonconformity or transgender identity." Before meeting with providers in this clinical setting, youth were instructed to complete this measure taking into account the perceived viewpoints of their parents/caregivers as a unit. During the clinical encounter, clinical providers then assessed nuances of parental support and rejection per reports by youth and parents (when present), including potentially discrepant perceptions of parental support. On average, the instruments of relevance for this study were completed within 16-20 min and were only available in English.

\section{Survey participants}

Participants were 341 gender-expansive youth, aged $12-24$ years $(M=15.6, S D=1.99)$, who were outpatients of an interdisciplinary gender-health clinic located within a pediatric academic medical center in a large city within the Midwestern United States (Table 1). Human subjects' approval for this study was obtained from the Institutional Review Board of the pediatric academic medical center from which all data were collected. Two-thirds of the samples was assigned female at birth (66.9\%), and the remainder was assigned male at birth (no youth were intersex). In terms of race, $65.7 \%$ of the youth were white/European American, $11.1 \%$ were Hispanic/Latinx, $4.4 \%$ were black/African American, and 3.8\% were Asian/Pacific Islander.

\section{Instruments}

General parental support. Four items from the MSPSS $^{28}$ that related to family support were adapted

Table 1. Participant Demographic Information

\begin{tabular}{lcc}
\hline & $n=341$ & $\%$ \\
\hline Age & $12-24$ years & \\
M & 15.6 & \\
SD & 1.99 & \\
Sex assigned at birth & & 66.9 \\
Female & 228 & 33.1 \\
Male & 113 & \\
Race/ethnicity & & 65.7 \\
White (non-Latinx) & 224 & 4.4 \\
Black/African American (non-Latinx) & 15 & 3.1 \\
Hispanic/Latinx & 38 & 10 \\
Asian/Pacific Islander & 13 & 5 \\
Multiracial/other & 34 & \\
Missing & 17 & 37 \\
Education level & & 1 \\
High school or less & 126 & 2.9 \\
Some college & 3 & 59.2 \\
Not currently in school & $10^{\mathrm{a}}$ & \\
Missing/unreported & 202 & \\
\hline
\end{tabular}

${ }^{a}$ Not currently in school for an unknown reason. 
and included to test for convergent validity. We adapted these MSPSS items by changing "family" to "parent(s)" on items such as "I can talk about my problems with my family." The MSPSS has demonstrated validity and reliability among LGBTQ+ youth $(\alpha=0.094){ }^{29}$ For the purpose of including these items along with the 17 items described above, we trimmed the original 7-point Likert scale of the MSPSS items (Very Strongly Disagree to Very Strongly Agree) to a 5 -point scale that matched the scale described above. Higher scores on these items were indicative of greater general parental support. The Cronbach's alpha of these four study items was 0.87 .

Nonaffirmation of gender identity. The nonaffirmation of Gender Identity subscale of the Gender Minority Stress and Resilience (GMSR-NA) $\mathrm{Scale}^{30}$ was included in the survey. We began collecting data on the GMSR $\sim 8$ months after launching our clinic and, therefore, the sample consisted of 261 respondents. While, the GMSR-NA was developed for individuals older than the age of 18 years, we chose to include the selected items due to their relevance to gender-expansive adolescents younger than the age of 18 years. We included the GMSR-NA to test for discriminant validity. The GMSR includes a six-item gender-related rejection subscale (Cronbach's alpha $=0.71$ ); however, this subscale was not used in this study as only one item within this rejection scale relating to family-based rejection. The GSMRNA subscale consists of six items that assess degree of nonaffirmation using a 5-point Likert response scale (Strongly Disagree to Strongly Agree). Sample items include "People don't respect my gender identity because of my appearance or body" and "I have to repeatedly explain my gender identity to people or correct the pronouns people use." Higher scores were indicative of greater nonaffirmation. The validity of this subscale (i.e., $\alpha=0.93$ ) has been recorded elsewhere, ${ }^{31}$ and the Cronbach's alpha of this study subscale was 0.86 .

\section{Statistical analysis}

Analysis occurred in three phases. First, we ran bivariate correlations of the 17 -item questionnaire to evaluate their suitability for factor analysis. Second, we explored the presence of any underlying factor structure from the 17 items using principal components analysis (PCA). We chose PCA due to its ability to prioritize the identification of a component/factor that accounts for the majority of variance contributed by various items. ${ }^{32}$ We estimated the number of components through a scree plot test, followed by a Monte Carlo parallel analysis (PA). To assist in the interpretation of potential factors, we performed oblimin rotation as it maximizes the loadings of items believed to be strongly interassociated to one factor. ${ }^{33}$ In the PA interpretation of potential factors, we were mindful to apply a 95th percentile criterion when assessing the first eigenvalue and a mean eigenvalue criterion when evaluating subsequent eigenvalues. ${ }^{34}$ This approach corrects for potential underfactoring by PA in conditions in which factors are hypothesized to be interrelated and when the first component's eigenvalue tends to be large. ${ }^{35,36}$ Last, we explored psychometric properties, including validity and reliability. To explore construct validity, we summed scores of significant components identified in PCA. We, then, conducted bivariate correlations between these component scores and summed scale scores from MSPSS and GMSR-NA to evaluate convergent and discriminant validity, respectively. Internal consistency was assessed using reliability analysis. All statistics were performed on SPSS version $24 .^{37}$

\section{Results}

Exploratory factor analysis

In assessing the suitability of data for factor analysis, an inspection of the correlation matrix revealed the presence of many coefficients above 0.3. However, three items, "My parent(s) encourage me to wear clothing and accessories consistent with my assigned sex at birth," "My parent(s)' religious beliefs help them be supportive of me," and "My parent(s) are afraid of the physical effects of gender transitioning" were poorly correlated (i.e., $r<0.3$ ) with other scale items, resulting in their removal. The remaining 14 items were subjected to PCA. The Kaiser-Meyer-Olkin value of these 14 items was 0.93 , exceeding the recommended value of 0.6 and Bartlett's test of sphericity reached statistical significance, supporting the factorability of the correlation matrix.

PCA revealed the presence of two components with eigenvalues exceeding 1 , explaining $56 \%$ and $7.6 \%$ of the variance, respectively (a total $63.6 \%$ variance explained) (Table 2). An inspection of the scree plot revealed a break coinciding with the second component. Subsequent Monte Carlo PA indicated both components had eigenvalues exceeding the corresponding 
Table 2. Comparison of Eigenvalues from Principal Components Analysis and Criterion Values from Parallel Analysis

\begin{tabular}{|c|c|c|c|c|}
\hline \multirow[b]{2}{*}{ Component } & \multicolumn{3}{|c|}{ Initial eigenvalues } & \multirow[b]{2}{*}{ PA criterion value } \\
\hline & Total & $\%$ of variance & Cumulative \% & \\
\hline 1 & 7.85 & 56 & 56 & 1.35 \\
\hline 2 & 1.06 (4.45 $5^{\mathrm{a}}$ mean criterion) & 7.6 & 63.6 & 1.27 \\
\hline
\end{tabular}

${ }^{a}$ Mean eigenvalue rule used for Component 2.

$\mathrm{PA}$, parallel analysis.

criterion values for a randomly generated data matrix of the same size (14 variables $\times 341$ respondents). The oblimin rotation solution revealed the presence of a simple structure, with both components showing a number of strong loadings and 12 variables loading substantially on one or the other component (Table 3). For the two cases in which variables simultaneously loaded onto two components (i.e., "My parent(s) are ashamed of me" and "My parent(s) have problems with my gender expression"), the component with the greatest variable strength retained the variable. In terms of interpretation, items related to perceived parental nonaffirmation loaded strongly on Component 1 and perceived parental acceptance on Component 2. There was a moderate inverse correlation between the two factors $(r=-0.65)$. The results of this analysis suggest the use of eight perceived parental nonaffirmation and six perceived parental acceptance items as separate scales (Table 4).
Reliability and validity

In terms of internal reliability, scales demonstrated sufficient internal consistency in subscale $1(\alpha=0.91)$, subscale $2(\alpha=0.88)$, and as a total scale $(\alpha=0.94)$. Convergent validity for subscale 1 was demonstrated in comparison with GMSR-NA items $(r=-0.29$, $p<0.01$ ), and discriminant validity for subscale 1 was demonstrated in comparison with MSPSS items $(r=-0.72, p<0.01)$. Convergent validity for subscale 2 was demonstrated in comparison with MSPSS items $(r=0.81, p<0.01)$, and discriminant validity for subscale 2 was demonstrated in comparison with GMSR-NA items $(r=-0.31, p<0.01)$.

\section{Discussion}

This study aimed to explore the potential underlying factor structure of a brief questionnaire assessing aspects of parental support in gender-expansive adolescents and young adults. The 17 items that we analyzed

Table 3. Pattern and Structure Matrix for Principal Components Analysis with Oblimin Rotation of the Two Factor Scale Items

\begin{tabular}{|c|c|c|c|c|c|}
\hline \multirow[b]{3}{*}{ Item } & \multirow{2}{*}{\multicolumn{2}{|c|}{$\begin{array}{c}\text { Pattern coefficients } \\
\text { Component }\end{array}$}} & \multirow{2}{*}{\multicolumn{2}{|c|}{$\begin{array}{c}\text { Structure coefficients } \\
\text { Component }\end{array}$}} & \multirow[b]{3}{*}{ Commonalities } \\
\hline & & & & & \\
\hline & 1 & 2 & 1 & 2 & \\
\hline 1. My parents are proud of me & & -0.607 & 0.570 & -0.721 & 0.537 \\
\hline 2. My parents are ashamed of me & 0.505 & -0.382 & 0.754 & -0.711 & 0.653 \\
\hline 3. My parents try to hide me & 0.658 & & 0.757 & -0.581 & 0.586 \\
\hline 4. I can be myself around my parents & & -0.729 & 0.557 & -0.782 & 0.616 \\
\hline 5. My parents advocate for my rights as a gender-expansive/trans* child & & -0.914 & 0.551 & -0.885 & 0.784 \\
\hline $\begin{array}{l}\text { 6. My parents protect me and defend me against others } \\
\text { prejudice against gender-expansive/trans* people }\end{array}$ & & -0.952 & 0.457 & -0.846 & 0.730 \\
\hline 7. My parents have problems with my gender expression & 0.421 & -0.466 & 0.725 & -0.741 & 0.651 \\
\hline $\begin{array}{l}\text { 8. My parents use rewards or treats to pressure me to live } \\
\text { as my sex assigned at birth }\end{array}$ & 0.846 & & 0.740 & -0.388 & 0.563 \\
\hline 9. I can talk to my parents about romantic relationships and dating & & -0.543 & 0.526 & -0.655 & 0.446 \\
\hline $\begin{array}{l}\text { 10. My parents worry about how my gender identity will } \\
\text { affect our family's image }\end{array}$ & 0.712 & & 0.821 & -0.631 & 0.690 \\
\hline $\begin{array}{l}\text { 11. My parents probably believe they are bad parents because } \\
\text { I am gender-expansive/trans* }\end{array}$ & 0.843 & & 0.850 & -0.560 & 0.722 \\
\hline $\begin{array}{l}\text { 12. My parents probably believe that I am gender-expansive/ } \\
\text { trans* because of something they did wrong }\end{array}$ & 0.839 & & 0.849 & -0.562 & 0.721 \\
\hline 13. My parents are supportive of my gender transition & 0.302 & -0.603 & 0.696 & -0.800 & 0.693 \\
\hline $\begin{array}{l}\text { 14. My parents are worried that my gender identity is a bad } \\
\text { influence on other kids in my family }\end{array}$ & 0.576 & & 0.703 & -0.570 & 0.515 \\
\hline
\end{tabular}




\begin{tabular}{|c|c|c|c|}
\hline No. & Item & $\begin{array}{c}\text { Perceived } \\
\text { parental } \\
\text { non-affirmation } \\
\text { (Component 1) }\end{array}$ & $\begin{array}{c}\text { Perceived } \\
\text { parental } \\
\text { acceptance } \\
\text { (Component 2) }\end{array}$ \\
\hline 1 & My parent(s) are proud of me & & $\mathrm{x}$ \\
\hline $2 \mathrm{R}$ & My parent(s) are ashamed of me & $\mathrm{x}$ & \\
\hline $3 R$ & My parent(s) try to hide me & $\mathrm{x}$ & \\
\hline 4 & I can be myself around my parent(s) & & $\mathrm{x}$ \\
\hline 5 & My parent(s) advocate for my rights as a gender-expansive/trans* child & & $\mathrm{x}$ \\
\hline 6 & My parent(s) protect me and defend me against others prejudice against gender-expansive/trans* people & & $\mathrm{x}$ \\
\hline $7 \mathrm{R}$ & My parent(s) have problems with my gender expression & $\mathrm{x}$ & \\
\hline $8 \mathrm{R}$ & My parent(s) use rewards or treats to pressure me to live as my sex assigned at birth & $\mathrm{x}$ & \\
\hline 9 & I can talk to my parent(s) about romantic relationships and dating & & $\mathrm{x}$ \\
\hline $10 \mathrm{R}$ & My parent(s) worry about how my gender identity will affect our family's image & $\mathrm{x}$ & \\
\hline $11 \mathrm{R}$ & My parent(s) probably believe they are bad parent(s) because I am gender-expansive/trans* & $\mathrm{x}$ & \\
\hline $12 \mathrm{R}$ & My parent(s) probably believe that I am gender-expansive/trans* because of something they did wrong & $\mathrm{x}$ & \\
\hline 13 & My parent(s) are supportive of my gender transition & & $\mathrm{x}$ \\
\hline 14R & My parent(s) are worried that my gender identity is a bad influence on other kids in my family & $\mathrm{x}$ & \\
\hline
\end{tabular}

Response scale: 1-strongly disagree, 2-disagree, 3-neither disagree nor agree, 4-agree, 5-strongly agree (" $\mathrm{R}$ " above, indicates a reversecoded item).

were developed by two SMEs with personal, clinical, and community-level experience with parent challenges to support and nonaffirmation on account of youth's gender-expansive identity and expression. Based on data from 341 gender-expansive adolescents and young adults, our analyses resulted in a 14-item scale that possessed two moderately correlated subscales indicative of (1) perceived parental nonaffirmation of gender expansiveness, and (2) perceived parental acceptance of gender expansiveness. Reliability and validity results provide support of this new questionnaire. Specifically, results indicated good convergent validity and discriminant validity for the two subscales. Convergent validity was supported by negative correlations between the perceived parental nonaffirmation scale and measures of general parental support, and positive correlations between the perceived parental acceptance scale and measures of general parental support. Regarding discriminant validity, results suggest adequate distinctions between the scale components and the GMSR-NA items as demonstrated by weak correlations between these constructs.

Consistent with previous research noted here, ${ }^{19,20}$ the emergence of these two separate components suggests that parental nonaffirmation and acceptance may be perceived to co-occur among some genderexpansive youth. This is an important distinction as some parents who may privately struggle to affirm their child's gender expansiveness or worry that a child's gender expansiveness may reflect negatively on the family, may still advocate for their child's rights and protect their child from prejudice. Thus, future research should explore the relative contribution of these distinct constructs of parental nonaffirmation and acceptance to risk and resilience among genderexpansive youth.

It is notable that three items originally generated by SMEs were removed after exploratory factor analysis and suggested that they were poorly correlated with other scale items. One of the items removed, "My parent(s) are afraid of the physical effects of gender transitioning," was rated highly by many youths in the study, suggesting that concerns related to medical intervention are present even among parents who are otherwise perceived to be supportive of their child's gender expansiveness and perceived to exhibit low levels of nonaffirmation.

\section{Limitations}

The findings of this study must be interpreted in light of several limitations. The sample represents a predominantly white sample of gender-expansive adolescents and young adults from a large metropolitan area, and study results may not be generalizable to racially/ ethnically diverse, gender-expansive youth and those in less urban areas. Data were collected from a clinical sample of youth seeking services from a single genderaffirming interdisciplinary service. These youth presented, in most cases, along with their parents/primary caregivers. Therefore, the sample may be less representative of gender-expansive youth whose parents/ caregivers are less likely to support their seeking gender-affirmative care. 
Limitations aside, the scale that resulted from our study has utility in clinical and research settings. This scale was completed based on adolescents' perceptions of all primary caregivers combined because it was administered immediately before a clinical encounter in which family support dynamics were qualitatively assessed. In the future, it is recommended that clinicians and researchers ask youth to complete a separate scale specific to each primary caregiver in their life, adapting the measure items to reduce the response burden (i.e., fatigue effects) for youth with more than two such figures in their life. Administering the scale in this manner may ensure that family dynamics related to support are better captured, including discrepant perceptions of support between parents/ caregivers. We do not believe that the approach taken in this study limited our ability to assess the construct of parental support nor the scale's internal validity and consistency.

\section{Conclusions}

Overall, the current study offers preliminary evidence of the factor structure, reliability and validity of the Parental Attitudes of Gender Expansiveness Scale for Youth (PAGES-Y). The PAGES-Y has research and clinical utility, demonstrating that it can assess experiences of parental acceptance and nonaffirmation simultaneously. Future research may use the PAGES- $Y$ to determine how these experiences may relate to medical and behavioral health outcomes, aid in identifying priorities for therapeutic intervention, and yield a more nuanced understanding of how parental attitudes of gender expansiveness contribute to risk and resilience among gender-expansive adolescents.

\section{Acknowledgments}

The authors wish to acknowledge the contributions of the young adult SME who wished to remain anonymous and Gia Chodzen, B.A. Research reported in this publication was supported, in part, by the Eunice Kennedy Shriver National Institute of Child Health \& Human Development of the National Institutes of Health (NIH) under Award Number R01HD082554. The content is solely the responsibility of the authors and does not necessarily represent the official views of the NIH.

\section{Author Disclosure Statement}

No competing financial interests exist.

\section{References}

1. Scholte $\mathrm{RH}$, van Lieshout $\mathrm{CF}$, van Aken MA. Perceived relational support in adolescence: dimensions, configurations, and adolescent adjustment. J Res Adolesc. 2001;11:71-94.

2. Claes L, Luyckx K, Baetens I, et al. Bullying and victimization, depressive mood, and non-suicidal self-injury in adolescents: the moderating role of parental support. J Child Fam Stud. 2015;24:3363.

3. Bontempo DE, D'Augelli AR. Effects of at-school victimization and sexual orientation on lesbian, gay, or bisexual youths' health risk behaviors. J Adolesc Health. 2002;30:364-374.

4. Garofalo R, Deleon J, Osmer E, et al. Overlooked, misunderstood and atrisk: exploring the lives and HIV risk of ethnic minority male-to-female transgender youth. J Adolesc Health. 2006;38:230-236.

5. D'Augelli AR, Grossman AH, Salter NP, et al. Predicting the suicide attempts of lesbian, gay, and bisexual youth. Suicide Life Threat Behav. 2005;35:646-660.

6. D'Augelli AR, Grossman AH, Starks MT. Childhood gender atypicality, victimization, and PTSD among lesbian, gay, and bisexual youth. J Interpers Violence. 2006;21:1462-1482.

7. D'Augelli AR, Pilkington NW, Hershberger SL. Incidence and mental health impact of sexual orientation victimization of lesbian, gay, and bisexual youths in high school. Sch Psychol Q. 2002;17:148-167.

8. Haas AP, Eliason M, Mays VM, et al. Suicide and suicide risk in lesbian, gay, bisexual, and transgender populations: review and recommendations. J Homosex. 2011;58:10-51.

9. Hershberger SL, D'Augelli AR. The impact of victimization on the mental health and suicidality of lesbian, gay, and bisexual youths. Dev Psychol. 1995;31:65-74.

10. Ryan C, Russell ST, Huebner D, et al. Family acceptance in adolescence and the health of LGBT young adults. Child Adolesc Psychiatr Nurs. 2010;23:205-213.

11. Travers R, Bauer G, Pyne J, et al. Impacts of parental support for trans youth: a report prepared for Children's Aid Society of Toronto and Delisle Youth Services. 2012. http://transpulseproject.ca/wp-content/uploads/2012/10/ Impacts-of-Strong-Parental-Support-for-Trans-Youth-vFINAL.pdf (last accessed July 15, 2015).

12. Wilson EC, Iverson E, Garofalo R, Belzer M. Parental support and condom use among transgender female youth. J Assoc Nurses AIDS Care. 2012;23:306-317.

13. McConnell EA, Birkett M, Mustanski B. Families matter: social support and mental health trajectories among lesbian, gay, bisexual, and transgender youth. J Adolesc Health. 2016;59:674-680.

14. Connolly MD, Zervos MJ, Barone CJ, et al. The mental health of transgender youth: advances in understanding. J Adolesc Health. 2016;59: 489-495.

15. Corliss $H$, Belzer M. An evaluation of service utilization among male to female transgender youth: qualitative study of a clinic-based sample. J LGBT Health Res. 2007;3:53-69.

16. Garofalo R, Deleon J, Osmer E, et al. Overlooked, misunderstood and atrisk: exploring the lives and HIV risk of ethnic minority male-to-female transgender youth. J Adolesc Health. 2006;38:230-236.

17. Grossman AH, D'Augelli AR. Transgender youth: invisible and vulnerable. J Homosex. 2006;51:111-128.

18. Wilson EC, Garofalo R, Harris DR, et al. Sexual risk taking among transgender male-to-female youths with different partner types. Am J Public Health. 2010;100:1500-1505.

19. Perrin EC, Cohen KM, Gold M, et al. Gay and lesbian issues in pediatric health care. Curr Probl Pediatr Adolesc Health Care. 2004;34:355-398.

20. Khaleque A, Rohner RP. Perceived parental acceptance-rejection and psychological adjustment: a meta-analysis of cross-cultural and intracultural studies. J Marriage Fam. 2002;64:54-64.

21. Hill DB, Menvielle E. "You have to give them a place where they feel protected and safe and loved": the views of parents who have gendervariant children and adolescents. J LGBT Youth. 2009;6:243-271.

22. Simons L, Schrager SM, Clark LF, et al. Parental support and mental health among transgender adolescents. J Adolesc Health. 2013;53: 791-793.

23. Le V, Arayasirikul S, Chean Y, et al. Types of social support and parental acceptance among transfemale youth and their impact on mental health, sexual debut, history of sex work and condomless anal intercourse. J Int AIDS Soc. 2016;19. 
24. Riley EA, Sitharthan G, Clemson L, Diamond M. Recognizing the needs of gender-variant children and their parents. Sex Educ. 2013;13:644-659.

25. Chen D, Hidalgo MA, Leibowitz $S$, et al. Multidisciplinary care for genderdiverse youth: a narrative review and unique model of gender-affirming care. Transgender Health. 2016;1:117-123.

26. Hidalgo MA, Ehrensaft D, Tishelman $A C$, et al. The gender affirmative model: what we know and what we aim to learn. Hum Dev. 2013;56:285-290.

27. Malpas J. Between pink and blue: a multi-dimensional family approach to gender nonconforming children and their families. Fam Process. 2011;50:453-470.

28. Zimet GD, Dahlem NW, Zimet SG, Farley GK. The multidimensional scale of perceived social support. J Pers Assess. 1988;52:30-41.

29. Ybarra ML, Mitchell KJ, Palmer NA, Reisner SL. Online social support as a buffer against online and offline peer and sexual victimization among US LGBT and non-LGBT youth. Child Abuse Negl. 2015;39:123-136.

30. Testa RJ, Habarth J, Peta J, et al. Development of the gender minority stress and resilience measure. Psychol Sex Orientat Gend Divers. 2014;2:65-77.

31. Fishbein $M$, Hennessy $M$, Kamb $M$, et al. Using intervention theory to model factors influencing behavior change. Project RESPECT. Eval Health Prof. 2001;24:363-384.

32. Rattray J, Jones MC. Essential elements of questionnaire design and development. J Clin Nurs. 2005;16:234-243.

33. Oppenheim AN. Questionnaire Design, Interviewing and Attitude Measurement. London: Pinter, 1992.

34. Crawford AV, Green SB, Levy R, et al. Evaluation of parallel analysis methods for determining the number of factors. Educ Psychol Meas. 2010;70:855-901.

35. Turner NE. The effect of common variance and structure pattern on random data eigenvalues: implications for the accuracy of parallel analysis. Educ Psychol Meas.1998;58:541-568.
36. Beauducel A. Problems with parallel analysis in data sets with oblique simple structure. Methods Psychol Res Online 2001. 2001;6:141-157.

37. IBM SPSS Statistics for Windows [computer program]. Version 24.0. Armonk, NY: IBM Corp., 2016.

Cite this article as: Hidalgo MA, Chen D, Garofalo R, Forbes C (2017) Perceived parental attitudes of gender expansiveness: development and preliminary factor structure of a self-report youth questionnaire Transgender Health 2:1, 180-187, DOI: 10.1089/trgh.2017.0036.

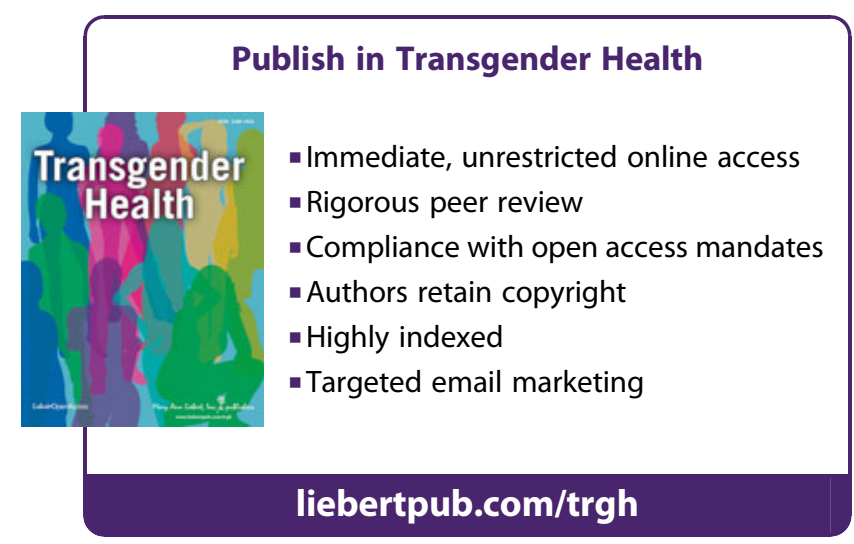

